\title{
Transtorno da conduta e comportamento anti-social
}

\author{
Isabel AS Bordina e David R Offord ${ }^{b}$
}

\begin{abstract}
${ }^{a}$ Departamento de Psiquiatria, Universidade Federal de São Paulo/Escola Paulista de Medicina (Unifesp/EPM) e Grupo Interdepartamental de Epidemiologia Clínica (Gridec), Unifesp/EPM. 'Divisão de Psiquiatria da Infância e Adolescência, McMaster University, Canadá e Centro de Estudos sobre Crianças em Situação de Risco e Centro Chedoke para a Criança e a Família, McMaster University, Canadá
\end{abstract}

\section{Introdução}

Certos comportamentos, como mentir e matar aula, podem ser observados no curso do desenvolvimento normal de crianças e adolescentes. Para diferenciar normalidade de psicopatologia, é importante verificar se esses comportamentos ocorrem esporadicamente e de modo isolado ou se constituem síndromes, representando um desvio do padrão de comportamento esperado para pessoas da mesma idade e sexo em determinada cultura.

A literatura internacional aborda o tema do comportamento anti-social sob diferentes pontos de vista, levando em conta os aspectos legais (criminologia) e psiquiátricos. Do ponto de vista legal, a delinqüência implica em comportamentos que transgridem as leis. No entanto, como nem todas as crianças ou jovens anti-sociais transgridem as leis, o termo delinqüente ficou restrito aos menores infratores (definição legal). Os atos anti-sociais relacionados aos transtornos psiquiátricos são mais abrangentes e se referem a comportamentos condenados pela sociedade, com ou sem transgressão das leis do Estado. ${ }^{1}$

Com base em critérios diagnósticos internacionais, como os da última edição do Diagnostic and Statistical Manual of Mental Disorders (DSM-IV²), observa-se que o comportamento antisocial persistente faz parte de alguns diagnósticos psiquiátricos. O transtorno da conduta (conduct disorder) e o transtorno desafiador de oposição (oppositional defiant disorder) são categorias diagnósticas usadas para crianças e adolescentes, enquanto o transtorno de personalidade anti-social (antisocial personality disorder) aplica-se aos indivíduos com 18 anos ou mais.

No presente artigo, serão apresentadas as principais características do transtorno da conduta, enfatizando seu diagnóstico, evolução e tratamento. Destacaremos os fatores associados ao comportamento anti-social na infância e adolescência com o objetivo de ampliar a visão do profissional de saúde mental sobre a família e a comunidade nas quais o paciente está inserido.

\section{Transtorno da conduta}

O transtorno da conduta é um dos transtornos psiquiátricos mais freqüentes na infância e um dos maiores motivos de encaminhamento ao psiquiatra infantil. ${ }^{3}$ Lembramos que o transtorno da conduta não deve ser confundido com o termo "distúrbio da conduta", utilizado no Brasil de forma muito abrangente e inespecífica para nomear problemas de saúde mental que causam incômodo no ambiente familiar e/ou escolar. Por exemplo, crianças e adolescentes desobedientes, com dificuldade para aceitar regras e limites e que desafiam a autoridade de pais ou professores costumam ser encaminhados aos serviços de saúde mental devido a "distúrbios da conduta". No entanto, os jovens que apresentam tais distúrbios nem sempre preenchem critérios para a categoria diagnóstica "transtorno da conduta". Portanto, o termo "distúrbio da conduta" não é apropriado para representar diagnósticos psiquiátricos.

No Canadá, o transtorno da conduta atinge 5,5\% dos indivíduos da população geral com idade entre 4-16 anos, com taxas variando de 1,8\% (meninas de 4-11 anos) a 10,4\% (meninos de 12-16 anos). O transtorno da conduta é mais freqüente no sexo masculino, independentemente da idade, e mais freqüente em crianças maiores (12-16 anos) comparadas às menores (4-11 anos), independentemente do sexo.,

\section{Diagnóstico}

$\mathrm{Na}$ base do transtorno da conduta está a tendência permanente para apresentar comportamentos que incomodam e perturbam, além do envolvimento em atividades perigosas e até mesmo ilegais. Esses jovens não aparentam sofrimento psíquico ou constrangimento com as próprias atitudes e não se importam em ferir os sentimentos das pessoas ou desrespeitar seus direitos. Portanto, seu comportamento apresenta maior impacto nos outros do que em si mesmo. Os comportamentos antisociais tendem a persistir, parecendo faltar a capacidade de aprender com as consequiências negativas dos próprios atos. ${ }^{1}$

O quadro clínico do transtorno da conduta é caracterizado por comportamento anti-social persistente com violação de normas sociais ou direitos individuais. Os critérios diagnósticos do DSMIV para transtorno da conduta incluem 15 possibilidades de comportamento anti-social: (1) freqüentemente persegue, atormenta, ameaça ou intimida os outros; (2) freqüentemente inicia lutas corporais; (3) já usou armas que podem causar ferimentos graves (pau, pedra, caco de vidro, faca, revólver); (4) foi cruel com as pessoas, ferindo-as fisicamente; (5) foi cruel com os animais, ferindo-os fisicamente; (6) roubou ou assaltou, confrontando a vítima; (7) submeteu alguém a atividade sexual forçada; (8) iniciou incêndio deliberadamente com a intenção de provocar sérios danos; (9) destruiu propriedade alheia deliberadamente (não pelo fogo); (10) arrombou e invadiu casa, prédio ou carro; (11) mente e engana para obter ganhos materiais ou favores ou para fugir de obrigações; (12) furtou objetos de valor; (13) frequientemente passa a noite fora, apesar da proibição dos pais (início antes dos 13 anos); (14) fugiu de casa pelo menos duas vezes, passando a noite fora, enquanto morava com os pais ou pais 
substitutos (ou fugiu de casa uma vez, ausentando-se por um longo período); e (15) falta na escola sem motivo, matando aulas freqüentemente (início antes dos 15 anos). Os critérios diagnósticos do DSM-IV para transtorno da conduta aplicam-se a indivíduos com idade inferior a 18 anos e requerem a presença de pelo menos três desses comportamentos nos últimos 12 meses e de pelo menos um comportamento anti-social nos últimos seis meses, trazendo limitações importantes do ponto de vista acadêmico, social ou ocupacional. ${ }^{2}$

Diagnósticos diferenciais incluem os transtornos reativos a situações de estresse e comportamento anti-social decorrente de quadros psicóticos (por exemplo, episódio maníaco). ${ }^{2}$ Crianças vítimas de violência doméstica podem apresentar comportamentos anti-sociais como reação a situações de estresse e adolescentes em episódio maníaco podem furtar, falsificar assinaturas em cheques ou provocar brigas com luta corporal em decorrência da exaltação do humor e não devido a transtorno da conduta.

\section{Curso e prognóstico}

Os sintomas do transtorno da conduta surgem no período compreendido entre o início da infância e a puberdade e podem persistir até a idade adulta. ${ }^{3}$ Quando iniciam-se antes dos 10 anos, observa-se com maior freqüência a presença de transtorno com déficit de atenção e hiperatividade (TDAH), comportamento agressivo, déficit intelectual, convulsões e comprometimento do sistema nervoso central devido a exposição a álcool/drogas no período pré-natal, infecções, uso de medicamentos, traumas cranianos, etc., além de antecedentes familiares positivos para hiperatividade e comportamento anti-social. ${ }^{6} \mathrm{O}$ início precoce indica maior gravidade do quadro com maior tendência a persistir ao longo da vida. ${ }^{6}$

$\mathrm{O}$ transtorno da conduta está freqüentemente associado a TDAH (43\% dos casos) e a transtornos das emoções (ansiedade, depressão, obsessão-compulsão; $33 \%$ dos casos). ${ }^{7}$ A comorbidade com o TDAH é mais comum na infância, envolvendo principalmente os meninos, enquanto a comorbidade com ansiedade e depressão é mais comum na adolescência, envolvendo principalmente as meninas após a puberdade.

Comportamentos anti-sociais mais graves (por exemplo, brigas com uso de armas, arrombamentos, assaltos) costumam ser antecedidos por comportamentos mais leves (por exemplo, mentir, enganar, matar aulas, furtar objetos de pouco valor) e, ao longo do tempo, observa-se o abuso de álcool/drogas, principalmente no sexo masculino ${ }^{8}$ e os quadros de ansiedade e depressão, principalmente no sexo feminino..$^{9,10}$

O transtorno da conduta está freqüentemente associado a baixo rendimento escolar e a problemas de relacionamento com colegas, trazendo limitações acadêmicas e sociais ao indivíduo. ${ }^{11,12}$ São frequientes os comportamentos de risco envolvendo atividades sexuais, uso de drogas e até mesmo tentativas de suicídio. $\mathrm{O}$ envolvimento com drogas e gangues pode iniciar o jovem na criminalidade. Na fase adulta, notam-se sérias conseqüências do comportamento anti-social, como discórdia conjugal, perda de empregos, criminalidade, prisão e morte prematura violenta. ${ }^{2,13,14}$

A persistência de comportamentos anti-sociais no decorrer da adolescência e da vida adulta encontra-se favorecida em determinadas circunstâncias: quando o transtorno da conduta tem início precoce; quando tipos variados de comportamentos anti-sociais estão presentes, incluindo os agressivos e violentos; quando os comportamentos anti-sociais são bastante freqüentes; quando são observados em diversos ambientes (por exemplo, familiar e escolar); e quando o transtorno da conduta está associado ao TDAH. ${ }^{15}$

Eventos de vida podem favorecer a persistência do comportamento anti-social na adolescência e idade adulta. $\mathrm{O}$ ambiente escolar, dependendo de suas características, pode incentivar ou desestimular o comportamento anti-social. Já a falta de emprego é uma situação de estresse que o estimula, enquanto o casamento harmonioso com pessoa sem alterações de comportamento tende a diminuí-lo. ${ }^{10}$

Ao mesmo tempo em que crianças com comportamento antisocial tendem a permanecer anti-sociais na idade adulta, adultos anti-sociais tendem a ter filhos com comportamento antisocial (pais servem de modelo aos filhos), estabelecendo-se um ciclo de difícil interrupção. ${ }^{16}$

\section{Fatores associados ao comportamento anti-social}

O comportamento anti-social de crianças e adolescentes tem sido atribuído a fatores constitucionais e ambientais. Historicamente, foi com o estabelecimento de clínicas vinculadas ao juizado de menores que profissionais de saúde mental puderam observar o desenvolvimento do comportamento anti-social na infância e adolescência. Ao constatar-se a grande freqüência de problemas familiares e sociais na história de vida dos delinqüentes juvenis, formulou-se a hipótese de uma reação às adversidades encontradas tanto no ambiente familiar como na comunidade. ${ }^{8}$

Segundo Winnicott, ${ }^{17}$ quando crianças sofrem privação afetiva, manifestam-se os comportamentos anti-sociais no lar ou numa esfera mais ampla. Do ponto de vista psicodinâmico, estes comportamentos demonstram esperança em obter algo bom que foi perdido, sendo a ausência de esperança a característica básica da criança que sofreu privação. $O$ jovem experimenta um impulso de busca do objeto, de alguém que possa encarregar-se de cuidar dele, esperando poder confiar num ambiente estável, capaz de suportar a tensão resultante do comportamento impulsivo. O ambiente é repetidamente testado em sua capacidade para suportar a agressão, tolerar o incômodo, impedir a destruição, preservando o objeto que é procurado e encontrado. ${ }^{17}$

São fatores associados a comportamento anti-social na infância: ser do sexo masculino, receber cuidados maternos e paternos inadequados, viver em meio à discórdia conjugal, ser criado por pais agressivos e violentos, ter mãe com problemas de saúde mental, residir em áreas urbanas e ter nível socioeconômico baixo. ${ }^{12,18,19}$

Alguns autores referem que a baixa renda associada ao comportamento anti-social da criança está relacionada à personalidade anti-social materna ${ }^{20,21}$ e à negligência por parte dos pais. ${ }^{22}$ De fato, pode-se supor que mães anti-sociais teriam maior dificuldade para atingir níveis de renda mais elevados, pois não manter-se-iam no emprego e teriam menor condição de manter um relacionamento estável com um marido ou companheiro que contribuísse com a renda familiar. Pais anti-sociais também são freqüentemente irresponsáveis e negligentes com seus 
filhos, deixando de alimentá-los adequadamente ou levá-los ao médico quando doentes. Além disso, adolescentes vivendo na pobreza e pouco valorizados pelos pais podem buscar reconhecimento pessoal e ascensão econômica através de atividades delinqüenciais grupais. ${ }^{23}$

Quanto à discórdia conjugal e a problemas mentais maternos, Shaw e Emery ${ }^{18}$ demonstraram que o conflito entre os pais e a depressão materna estavam associados a comportamentos agressivos e anti-sociais em escolares. No entanto, é preciso considerar a contribuição da criança para a qualidade do relacionamento entre pais e filhos, pois crianças difíceis de lidar, desobedientes e agressivas favorecem a desorganização do ambiente familiar e o desequilíbrio de um relacionamento conjugal mais frágil. ${ }^{1}$

Quanto ao ambiente familiar agressivo e violento, não se pode deixar de mencionar a influência da violência doméstica e do abuso físico sobre o comportamento anti-social na infância. Taxa elevada de comportamento anti-social (21\%) foi observada em filhos (idade escolar) de mulheres espancadas. ${ }^{24} \mathrm{Em}$ Khartoum, Sudão, crianças submetidas à punição corporal grave (corda ou vara) apresentaram mais problemas de comportamento $(40,2 \%)$ que crianças punidas somente com palmadas $(24,6 \%) .{ }^{25}$ Estudos que avaliaram os efeitos do abuso físico a longo prazo demonstraram que indivíduos que sofreram abuso ou negligência na infância tiveram maior probabilidade de cometer crimes. ${ }^{26,27}$ No entanto, a grande maioria das crianças que sofreram abuso (74\%) ou negligência (90\%) não se tornaram delinqüentes, nem cometeram crimes violentos. ${ }^{27}$

Fatores genéticos e neurofisiológicos também podem estar envolvidos no desenvolvimento do comportamento anti-social. ${ }^{1}$ Mednik et al $^{28}$ relataram maior taxa de criminalidade nos pais biológicos que nos pais adotivos de indivíduos com antecedentes criminais, formulando a hipótese de uma predisposição biológica para o comportamento anti-social. A influência genética é mais evidente nos casos acompanhados de hiperatividade e pode ser responsável pela maior vulnerabilidade do indivíduo aos eventos de vida e ao estresse. ${ }^{29}$ No entanto, o papel dos fatores genéticos no transtorno da conduta ainda precisa ser melhor esclarecido.

Finalmente, há indícios de diferenças nos fatores de risco para o comportamento anti-social segundo o sexo do indivíduo. ${ }^{15,29}$ Em levantamento populacional realizado no Canadá, envolvendo 1.651 indivíduos com 16 a 24 anos, verificou-se que além da presença de comportamentos anti-sociais antes dos 15 anos, outros fatores foram considerados de risco para comportamento anti-social na adolescência e início da vida adulta. Para os homens, foi fator de risco o fato de ter convivido na infância com pais com problemas de saúde mental (depressão, mania, episódios psicóticos), enquanto para as mulheres, destacaram-se o abuso sexual na infância e o fato de ter sido criada por pais com comportamento anti-social ou abuso de álcool/drogas. ${ }^{15}$

\section{Tratamento}

Os tratamentos citados na literatura são bastante variados, incluindo intervenções junto à família e à escola (por exemplo, psicoterapia familiar e individual, orientação de pais, comuni- dades terapêuticas e treinamento de pais e professores em técnicas comportamentais). Apesar de nenhum deles ser muito eficaz, principalmente como intervenção isolada, quanto mais precocemente iniciados e quanto mais jovem o paciente, melhores os resultados obtidos. ${ }^{29}$ Salienta-se a importância das intervenções concomitantes e complementares a longo prazo.

$\mathrm{Na}$ faixa etária dos três aos oito anos, alguns sintomas do transtorno desafiador de oposição (por exemplo, irrita-se com facilidade, recusa-se a cumprir regras ou atender solicitações dos adultos, perturba as pessoas deliberadamente) ou do transtorno da conduta (por exemplo, fere animais, furta) costumam ser identificados, merecendo ações preventivas junto à criança e aos seus pais e professores. Muitas vezes, o foco do problema está no conflito entre pais e filhos. Outras vezes, os pais estão demasiadamente envolvidos com problemas pessoais e necessitam de apoio. Alguns pais precisam de ajuda para estabelecer limites e escolher métodos mais apropriados para educar os filhos. O contato com a escola também pode ser útil para resolver conflitos entre professores e alunos e ajudar os professores a encontrar maneiras mais adequadas de lidar com as dificuldades da criança.

Quanto mais jovem o paciente e menos graves os sintomas, maior a probabilidade do indivíduo se beneficiar de uma psicoterapia. Quando trata-se de adolescente que já cometeu delitos, observa-se maior resistência à psicoterapia, podendo ser útil o envolvimento com profissionais especializados no manejo de jovens anti-sociais através de oficinas de artes, música e esportes. Nessas oficinas, o adolescente tem a oportunidade de estabelecer vínculo afetivo com os profissionais responsáveis pelas atividades, tomando-os como modelo, além de perceber-se capaz de criar, o que favorece o desenvolvimento da auto-estima. Sempre que possível, a família dos pacientes deve ser incluída no processo terapêutico, lembrando que muitas vezes os pais necessitam de tratamento psiquiátrico (por exemplo, abuso de drogas).

O tratamento com psicofármacos faz-se necessário em algumas situações nas quais os sintomas-alvo (por exemplo, idéias paranóides associadas à agressividade, convulsões) ou outros transtornos psiquiátricos (por exemplo, TDAH, depressão) estão presentes. Recomendamos cautela no uso de neurolépticos para o tratamento da agressividade, pois os riscos podem superar os benefícios. ${ }^{6}$

A hospitalização está indicada em casos de risco iminente para o paciente (por exemplo, suicídio, auto-agressão) ou para os demais (por exemplo, homicídio). Sempre que possível, optar por intervenções menos restritivas (por exemplo, hospital-dia). ${ }^{6}$

Em nosso meio, muitas vezes não dispomos dos recursos necessários para o tratamento da criança ou adolescente com comportamento anti-social. Quando esses recursos existem, nem sempre as famílias têm condições de comparecer ao serviço na freqüência recomendada. O profissional de saúde mental pode ser útil estabelecendo prioridades entre as diversas condutas terapêuticas possíveis e recomendando ao paciente aquela que julgar mais imprescindível.

\section{Conclusão}

Comportamentos anti-sociais são freqüentemente observados no período da adolescência como sintomas isolados e transitóri- 
os. Porém, estes podem surgir precocemente na infância e persistir ao longo da vida, constituindo quadros psiquiátricos de difícil tratamento. Fatores individuais, familiares e sociais estão implicados no desenvolvimento e na persistência do comportamento anti-social, interagindo de forma complexa e ainda pouco esclarecida. Como o comportamento anti-social torna-se mais estável e menos modificável ao longo do tempo, ${ }^{30}$ crianças e adolescentes com transtorno da conduta precisam ser identificados o mais cedo possível para que tenham maior oportunidade de beneficiar-se de intervenções terapêuticas e ações preventi- vas. O tratamento mais efetivo envolve a combinação de diferentes condutas junto à criança/adolescente, à família e à escola. Quando não é possível o acesso a intervenções complementares, o profissional de saúde mental deve identificar a conduta terapêutica prioritária em cada caso específico.

Fonte de financiamento: Capes (Proj. 1526/93; Proc. 1576/ 93-4).

\section{Referências}

1. Earls F. Oppositional-defiant and conduct disorders. In: Rutter M, Taylor E, Hersov LA, editors. Child and adolescent psychiatry: modern approaches. Oxford: Blackwell Scientific Publications; 1994. p. 308-29.

2. American Psychiatric Association (APA). Diagnostic and statistical manual of mental disorders. 4th ed. Washington (DC): APA; 1994.

3. Robins LN. Conduct disorders. J Child Psychol Psychiatry 1991;32:193-212.

4. Offord DR, Boyle MH, Fleming JE, Blum HM, Rae-Grant NI. Ontario child health study: summary of selected results. Can J Psychiatry 1989;34:483-91.

5. Offord DR, Boyle MH, Racine YA. The epidemiology of antisocial behavior in childhood and adolescence. In: Pepler D, Rubin KH, editors. The development and treatment of childhood aggression. New York: Lawrence Erlbaum; 1991. p. 31-54.

6. American Academy of Child and Adolescent Psychiatry (AACAP). Practice parameters for the assessment and treatment of children and adolescents with conduct disorder. J Am Acad Child Adolesc Psychiatry 1997;36(10 Suppl):122S-39S.

7. Offord DR, Boyle MH, Racine YA, Fleming JE, Cadman DT, Blum HM, et al. Outcome, prognosis, and risk in a longitudinal follow-up study. J Am Acad Child Adolesc Psychiatry 1992;31(5):916-23.

8. Loeber R, Dishion T. Early predictors of male delinquency: a review. Psychol Bull 1983;94:68-99.

9. Robins LN. The consequences of conduct disorder in girls. In: Olweus D, Block J, Radle-Yarrow, editors. The development of antisocial and prosocial behavior: research, theories and issues. New York: Academic Press; 1986.

10. Rutter M. Adolescence as a transition period: continuities and discontinuities in conduct disorder. J Adolesc Health 1992;13:451-60.

11. Hawkins JD, Lishner DM. Schooling and delinquency. In: Johnson $\mathrm{EH}$, editor. Handbook of crime and delinquency prevention. New York: Guilford Press; 1987. p. 179-221.

12. Offord DR. Conduct disorder: risk factors and prevention. In: Shaffer D, Philips I, Enzer NB, editors. Prevention of mental disorders, alcohol and other drug use. Rockville (MD): U.S. Department of Health and Human Services; 1989. p. 273-307.

13. Robins LN, McEvoy L. Conduct problems as predictors of substance abuse. In: Robins LN, Rutter M, editors. Straight and devious pathways from childhood to adulthood. Cambridge: Cambridge University Press; 1990. p. 182-204.

14. Zoccolillo M. Co-occurence of conduct disorder and its adult outcomes with depressive and anxiety disorders: a review. J Am Acad Child Adolesc Psychiatry 1992;31(3):547-56.
15. Bordin IAS. Fatores de risco para comportamento anti-social na adolescência e início da vida adulta [dissertação de tese de doutorado]. São Paulo (SP): Universidade Federal de São Paulo/Escola Paulista de Medicina; 1996.

16. Farrington DP. The twelfth Jack Tizard memorial lecture - The development of offending and antisocial behaviour from childhood: key findings from the Cambridge study in delinquent development. J Child Psychol Psychiatry 1995;360(6):929-64.

17. Winnicott DW. Privação e delinquiência. São Paulo: Martins Fontes; 1994.

18. Shaw DS, Emery RE. Chronic family adversity and school-age children's adjustment. J Am Acad Child Adolesc Psychiatry 1988;27(2):200-6.

19. Loeber R. Development and risk factors of juvenile antisocial behavior and delinquency. Clin Psychol Rev 1990;10:1-41.

20. Frick PJ, Lahey BB, Hartdagen S, Hynd GW. Conduct problems in boys: relations to maternal personality, marital satisfaction, and socioeconomic status. J Clin Child Psychol 1989;18(2):114-20.

21. Lahey BB, Russo MF, Walker JL, Piacentini JC. Personality characteristics of the mothers of children with disruptive behavior disorders. J Consult Clin Psychol 1989;57(4):512-5.

22. Kolvin FJ, Miller JW, Fleeting M, Kolvin PA. Social and parenting factors affecting criminal-offence rates: findings from the Newcastle thousand family study (1947-1980). Br J Psychiatry 1988;152:80-90.

23. Anolik SA. Family influences upon delinquency: biosocial and psychosocial perspectives. Adolescence 1983;18(71):489-98.

24. Wildin SR, Williamson WD, Wilson GS. Children of battered women: developmental and learning profiles. Clin Pediatr 1991;30(5):299-304.

25. Rahim SIA, Cederblad M. Effects of rapid urbanization on child behavior and health in a part of Khartoum, Sudan: II-Psychosocial influences on behaviour. Soc Sci Med 1986;22(7):723-30.

26. Lamphear VS. The impact of maltreatment on children's psychosocial adjustment: a review of the research. Child Abuse Negl 1989;9:25163.

27. Widom CS. The cycle of violence. Science 1989;244:160-6.

28. Mednick SA, Moffit TE, Pollock V, Talovic S, Gabvielli W. The inheritance of human deviance. In: Olweus D, Yarrow MR, editors. Development of antisocial and prosocial behavior. New York: Academic Press; 1986.

29. Rutter M, Giller H, Hagell A, editors. Antisocial behavior by young people. Cambridge: Cambridge University Press; 1998.

30. Loeber R. Antisocial behavior: more enduring than changeable? J Am Acad Child Adolesc Psychiatry 1991;30(3):393-7.

Correspondência: Isabel A. S. Bordin

Grupo Interdepartamental de Epidemiologia Clínica (Gridec) da Universidade Federal de São Paulo/Escola Paulista de Medicina Rua Botucatu, 685 - 04023-062 São Paulo, SP - Fax: (0xx11) 5575-6427 - E-mail: bordin@ psiquiatria.epm.br 